\title{
Globally Adaptive Decentralized Control of Robot Manipulators
}

\author{
$\mathrm{Su}-\mathrm{Hau} \mathrm{Hsu}^{1}$ and $\mathrm{Li}-\mathrm{Chen} \mathrm{Fu}^{1,2}$ \\ 'Dept. of Electrical Engineering \\ ${ }^{2}$ Dept. of Computer Science \& Information Engineering \\ National Taiwan University, Taipei, Taiwan.
}

TEL: 886-2-23622209

FAX: 886-2-23622209

E-mail: lichen@ccms.ntu.edu.tw

\begin{abstract}
In this paper, we develop a new adaptive decentralized controller of robot manipulator for trajectory tracking. With the nonlinear control terms, the closed-loop system is globally stable. Furthermore, the adaptive decentralized controller is considered with a $\sigma$-modification term such that it becomes robust to the noise in practical implementation. Finally, a numerical study is provided to verify the effectiveness of the proposed scheme.
\end{abstract}

Keywords: Decentralized control, adaptive control, robot manipulators, global stability, $\sigma$ modification.

\section{Introduction}

The control of a robotic manipulator is especially challenging due to the inherent high non-linearity in its dynamics. In a practical situation, the inevitable uncertainty in the underlying maniputator model, say, payload change, adds additional difficulty into the control task. Although significant achievements, marked by the development of centralized adaptive and robust control schemes, have been made to improve the tracking performance of robots, the decentralized controller structure is still adopted by the majority of modern robots in favor of its computation simplicity and low-cost hardware setup. Therefore, how to best improve the tracking performance of robots through decentralized control is still an interesting research topic that attracts great attention from robotic community.

For manipulator tracking tasks, decentralized ap- proaches are not that straightforward since the overall system can not be decomposed into subsystems whose states and control inputs are totally decoupled from one another because of the inherent coupling such as moment of inertia, Coriolis force, etc. In the recent researches, several attempts, e.g. by Fu [2], Liu [5], and Tang et al. [7], have been made for the so-called adaptive independent joint control (IJC) or adaptive decentralized control so that a separate actuator taking feedback only from that particular joint is responsible for the joint control. Following the work in Hsu and Fu [3], a globally adaptive decentralized control with the $\sigma$ modification term [Ioannou, and Sun, 4] is proposed such that the overall scheme becomes robust to the noise in practical implementation.

This paper is organized as follows. In Section 2, the problem is formulated for a general rigid $n$-link openchain manipulator. Section 3 proposes a new adaptive decentralized nonlinear control law and applies it to the 
robotic manipulators as described in the previous section. To illustrate the performance of the controller, a numerical simulation example is provided in Section 4. Finally, some conclusions are given in Section 5.

\section{Problem Statement}

For a general $n$-link rigid manipulator, its dynamic model can be derived by using the Lagrangian-Eiuler approach and can be expressed in a symbolic form as [6]:

$$
M(q) \ddot{q}+C(q, \dot{q}) \dot{q}+g(q)=\tau+d(t, q, \dot{q})
$$

where $q, \dot{q} \in \mathrm{R}^{n}$ are the joint configuration and the joint velocity of the manipulator, respectively, $\tau \in \mathrm{F}^{n}$ is the torque input, $M(q): \mathbf{R}^{n} \rightarrow \mathrm{R}^{n \times n}$ is the manipulator inertia matrix, $C(q, \dot{q}) \dot{q}: \mathrm{R}^{n} \times \mathrm{R}^{n} \rightarrow \mathrm{R}^{n}$ is the vector of Coriolis and centrifugal force, $g(q): \mathrm{R}^{n} \rightarrow \mathrm{R}^{n}$ is the vector of gravitational generalized force, and $d(t, q, \dot{q}):[0, \infty) \times \mathrm{R}^{n} \times$ $\mathrm{R}^{n} \rightarrow \mathrm{R}^{\boldsymbol{n}}$ is the input disturbance vector. This dynamic model has the following properties that will be used in the controller design [6]:

Property 1: The inertia matrix $M$ is a symmetric and positive definite matrix, which satisfies

$$
\mu_{m} I \leq M \leq \mu_{M} I, \quad \forall q \in \mathrm{R}^{n}
$$

for some bounded constants $\mu_{m}, \mu_{M}>0$.

Property 2: The matrix $C$ satisfies

$$
\|C\| \leq \mu_{c}\|\dot{q}\|, \quad \forall\left(q^{\mathrm{T}}, \dot{q}^{\mathrm{T}}\right)^{\mathrm{T}} \in \mathrm{R}^{2 n}
$$

for some bounded constants $\mu_{c}>0$.

Property 3: $\dot{M}-2 C$ is a skew-symmetric matrix, i.e.,

$$
z^{\mathrm{T}}(\dot{M}-2 C) z=0 \quad \forall z \in \mathrm{R}^{n} .
$$

Property 4: The gravity vector $g$ satisfies

$$
\|g\| \leq \mu_{G}, \quad \forall q \in \mathbf{R}^{n}
$$

for some bounded constant $\mu_{G}>0$.

Now let $g_{d}(t)$ denote the desired position trajectory for tracking which is generally chosen twice differentiable to guarantee smoothness of the motion. Let $\tau^{\mathrm{T}}:=\left[\tau_{1}, \ldots\right.$, $\tau_{n}$ ]. If the $i$ th element of the control input for $i=1, \ldots, n$, is only a function of joint configuration and velocity of the $i$ th joint, the control input $\tau$ is called a decientralized controller. In this study, a decentralized control scheme is to be developed such that $q(t) \rightarrow q_{d}(t)$ and $\dot{q}(t) \rightarrow \dot{q}_{d}(t)$ as $t \rightarrow \infty$

\section{Controller Design}

In this section, an adaptive decentralized control schemes will be developed for the control of a general $n$-link rigid manipulator. The following error signals are defined as $e=q-q_{d}$ and $s \equiv \dot{e}+\Lambda e$ where the feedback gain matrix $\Lambda$ is a positive definite matrix. Now the $d y-$ namics defined by the signals $e$ and $s$ can be derived as

$$
\begin{gathered}
\dot{e}=-\Lambda e+s \\
M(q) \dot{s}=-C(q, \dot{q}) s+\tau-v(t, q, \dot{q})
\end{gathered}
$$

where

$$
\begin{aligned}
v(t, q, \dot{q}) & =M(q)\left(\ddot{q}_{d}+\Lambda \dot{e}\right)+C(q, \dot{q})\left(\dot{q}_{d}+\Lambda e\right) \\
& +g(q)-d(t, q, \dot{q})
\end{aligned}
$$

behaves as the interconnection term. In the following, without loss of generality, several technical assumptions are made to pose the problem in a tractable manner.

Assumption 1: The feedback gain matrix $\Lambda$ are diagonal positive definite matrices; that is, $\Lambda=\operatorname{diag}\left(\lambda_{1}, \ldots, \lambda_{n}\right)>0$ for $\lambda_{i}>0, i=1, \cdots, n$.

Assumption 2: The desired position trajectory $q_{d}(t)$ and the time derivatives of $q_{\delta}(t), \dot{q}_{d}(t)$ are all bounded time-varying signals.

Assumption 3: The input disturbance $d=\left[d_{1}, \ldots, d_{n}\right]^{\mathrm{T}}$ is upper bounded as follows:

$$
\left|d_{i}(t, q, \dot{q})\right| \leq d_{i_{-} 1}+d_{i_{-} 2}\left|q_{i}\right|+d_{i_{-} 3}\left|\dot{q}_{i}\right|
$$

where $d_{i_{-} 1}, d_{i_{-} 2}, d_{i_{3} 3}$ are non-negative constants for $i=$ $1, \ldots, n$.

Before the claimed decentralized control law is designed, two useful lemmas should be derived first. In this study, we start with adopting the truncated $L_{\infty}$ norm defined as

$$
\|x\|_{T, \infty} \equiv \max _{1 \leq i \leq n} \sup _{i \in\{0, T]}\left|x_{i}(t)\right|
$$

where $T \in[0, \infty)$ for all real vector-valued functions $x \in$ $C^{n}[0, \infty)$. Now, the following lemma will provide us use- 
ful inequalities to develop the control scherne in this study.

Lemma 1: If there exists a constant $T>0$ such that $\|s\|_{T, \infty}$ exists, then there are positive constants $\alpha_{1}, \alpha_{2}, \alpha_{3}$, and $\alpha_{4}$ such that

$$
\begin{aligned}
& \|e(t)\| \leq \alpha_{1}\left\|e_{0}\right\|+\alpha_{2}\|s\|_{T, \infty} \\
& \|\dot{e}(t)\| \leq \alpha_{3}\left\|e_{0}\right\|+\alpha_{4}\|s\|_{T,-}
\end{aligned}
$$

for $t \in[0, T]$ with $T \in[0, \infty)$ where $e_{0}=e(0)$ is the initial condition of $e$, and positive constants $\beta_{1}, \beta_{2}$, and $\beta_{3}$ are such that

$$
\|v(t, q, \dot{q})\| \leq \beta_{1}+\beta_{2}\|s\|_{T, \infty}+\beta_{3}\|s\|_{T, \infty}^{2}
$$

for $t \in[0, T]$ with $T \in[0, \infty)$.

Proof: Consider the linear system defined by $\dot{e}=-\Lambda e+s, e(0)=e_{0}$. Since the matrix $-\Lambda$ is Hurwitz, we obtain (10) for $t \in[0, T]$ where $\alpha_{1}$ and $\alpha_{2}$ are positive parameters. Furthermore, according to the definition of the linear systems, we then obtain (11) for $t \in[0, T]$ where $\alpha_{3}$ and $\alpha_{4}$ are positive parameters. Next, according to the definition of the interconnection term $v$ in (7), after applying the aforementioned properties of the robotic manipulator and Assumption 3, we have (12) for $t \in[0, T]$, where $\beta_{1}, \beta_{2}$, and $\beta_{3}$ are positive parameters.

The Consider another control law $\tau^{\top}=\left[\tau_{1}, \ldots, \tau_{n}\right]$ given as follows

$$
\tau_{i}=\left\{\begin{array}{l}
-\frac{1}{\varepsilon_{i}} \hat{\theta}_{i_{-}-1}^{2} s_{i}-\theta_{i_{-} 2} s_{i}-\theta_{i_{-} 3} s_{i}^{3} \text { if } \hat{\theta}_{i_{-} 1}\left|s_{i}\right| \leq \varepsilon_{i} \\
-\hat{\theta}_{i_{-} l} \operatorname{sgn}\left(s_{i}\right)-\theta_{i_{-} 2} s_{i}-\theta_{i_{-} 3} s_{i}^{3} \text { if } \hat{\theta}_{i_{-} 1}\left|s_{i}\right|>\varepsilon_{i}
\end{array}\right.
$$

for $i=1, \ldots, n$, where the pararneters $\hat{\theta}_{i_{-} 1}, i=1, \ldots, n$, need to be adjusted on line, and $\theta_{i_{-} j}>0$ for $i=1, \ldots, n$, and $j=2,3$, and $\operatorname{sgn}(\cdot)$ denotes the sign function as follows

$$
\operatorname{sgn}(x)=\left\{\begin{array}{rr}
+1 & x>0 \\
0 & x=0 \\
-1 & x<0
\end{array}\right.
$$

Furthermore, the auxiliary signals $\varepsilon_{i}, i=1, \ldots, n$, satisfy

$$
\dot{\varepsilon}_{i}=-p_{i} \varepsilon_{i}, \quad \varepsilon_{i}(0)>0 \text { and } p_{i}>0
$$

which is used as the time-varying boundary layer. The more discussion on (13)-(15) is provided in [3]. With the control law (13)-(15), the closed-loop system becomes differential equations with discontinuous right hand sides [Filippov, 1]. A considerable amount of works has been carried out to deal with situations like this. Here, we base our results for these differential equations on Filippov's concept. Now, consider an adaptive law as follows:

$$
\dot{\hat{\theta}}_{i_{-1}}=\gamma_{i_{-} 1}\left(\left|s_{i}\right|-\sigma \hat{\theta}_{i_{-} 1}\right)
$$

where $\hat{\theta}_{i_{-1}}(0) \geq 0$ and the adaptive gains $\gamma_{i_{-}}>0, i=1$, $\ldots, n$, and the $\sigma$-modification constant $\sigma>0$. Due to the numerical noise caused by convergence of $\varepsilon$ to zero, the $\sigma$-modification term is adopted here. Note that the overall control scheme (13)-(16) is apparently in a decentralized structure.

Now, the following theorem is the main result in this study.

Theorem 2 (Globally Adaptive Decentralized Control Scheme with o-Modification): Under Assumption 1 3, consider the error dynamics of the robotic manipulator with the control law (13)-(15) as well as the adaptive law (16) defined above. Then all signal are bounded, and, furthermore, the position tracking error $e(t)$ will converge to a residue set whose size can be reduced by use of larger $\theta_{2, \min }$, and $\gamma_{1, \min }$, where $\theta_{2, \min }=\min \left\{\theta_{2_{-} 1}, \ldots, \theta_{2_{-} n}\right\}$, and $\gamma_{1, \text { min }}=\min \left\{\gamma_{1_{-} 1}, \ldots, \gamma_{n_{-} 1}\right\}$.

Proof: The following two steps guarantee the proof.

Step 1: Prove the signal $s(t)$ is ultimately bounded. Consider the Lyapunov-like function

$$
V(t)=\frac{1}{2} s^{\mathrm{T}} M s .
$$

Assume that, for some finite $T>0$, there exists $l_{1}>0$ such that $\sup _{i \in[0, T]}\|s(t)\|_{2} \leq l_{1}$. After taking the time derivative of $V$ along $s(t)$ for $t \in[0, T]$, the following two different cases will be obtained:

$$
\text { Case 1: } \hat{\theta}_{i_{-} \mid}\left|s_{i}\right| \leq \varepsilon_{i} \text { for } t \in[0, T] \text {. }
$$

$$
\begin{aligned}
\dot{V} \leq & -\sum_{i=1}^{n} \frac{1}{\hat{\varepsilon}_{i}} \hat{\theta}_{i_{-}}^{2} s_{i}^{2}-\theta_{2, \min }\|s\|_{2}^{2}-\frac{1}{n} \theta_{3, \min }\|s\|_{2}^{4} \\
& +\|s\|_{2}\left(\beta_{1}+\beta_{2} l_{1}+\beta_{3} l_{1}^{2}\right)
\end{aligned}
$$


Case 2: $\hat{\theta}_{i_{-} I}\left|s_{i}\right|>\varepsilon_{i}$ for $t \in[0, T]$

$$
\begin{aligned}
\dot{V} & \leq-\sum_{i=1}^{n} \hat{\theta}_{i_{-}}\left|s_{i}\right|-\theta_{2, \min }\|s\|_{2}^{2}-\frac{1}{n} \theta_{3, \min }\|s\|_{2}^{4} \\
& +\|s\|_{2}\left(\beta_{1}+\beta_{2} l_{1}+\beta_{3} l_{1}^{2}\right)
\end{aligned}
$$

From both Case 1 and 2, we can conclude that

$$
\dot{V} \leq-\theta_{2, \min }\|s\|_{2}^{2}
$$

for $t \in[0, T]$ for $\|s\|_{2} \geq\left(\frac{n\left(\beta_{3}+\beta_{2} t_{1}+\beta, t_{1}^{2}\right)}{\theta_{3, w}}\right)^{1 / 3}$, which implies that there exists $l_{2}>0$ such that, for $t \in[0, T]$, $\|s\|_{2} \leq l_{2}<l_{1}$ if $l_{1}$ is sufficiently large. As such, we can again conclude that $s(t)$ is ultimately bounded, i.e., there exists a finite $l_{3}>0$ such that $l_{3} \equiv \sup _{\mathrm{re}[0, \infty)}\|s(t)\|_{2}$.

Step 2: Prove all signals are bounded and the signal $s \rightarrow 0$ as $t \rightarrow \infty$. Now consider another Lyapunov-like function

$$
V(t)=\frac{1}{2} s^{\top} M s+\sum_{i=1}^{n}\left[\frac{1}{2} \gamma_{i_{\sim} 1}^{-1}\left(\hat{\theta}_{i_{-} 1}-\theta_{i_{-} 1}^{*}\right)^{2}+p_{i}^{-1} \varepsilon_{i}\right]
$$

where $\theta_{i, 1}^{*}, i=1, \ldots, n$, are the desirable but unknown parameters. After taking the time derivative of $V$ along the solution trajectories of the closed-loop system, the following two different cases will be obtained:

Case 1: $\hat{\theta}_{i_{-} 1}\left|s_{i}\right| \leq \varepsilon_{i}$ for $t \in[0, \infty)$.

$$
\begin{aligned}
\dot{V} \leq & -\sum_{i=1}^{n} \frac{1}{\varepsilon_{i}} \hat{\theta}_{i_{-}}^{2} s_{i}^{2}-\theta_{2, \min }\|s\|_{2}^{2}-\frac{1}{n} \theta_{3, \min }\|s\|_{2}^{4} \\
& +\|s\|_{2}\left(\beta_{1}+\beta_{2} l_{3}+\beta_{3} l_{3}^{2}\right)-\frac{1}{2} \sigma \sum_{i=1}^{n}\left(\hat{\theta}_{i_{-}}-\theta_{i, 1}^{*}\right)^{2} \\
& +\frac{1}{2} \sigma \sum_{i=1}^{n} \theta_{i_{-1}}^{*}-\theta_{1, \min }^{*}\|s\|_{2}
\end{aligned}
$$

Case 2: $\hat{\theta}_{i_{-} l}\left|s_{i}\right|>\varepsilon_{i}$ for $t \in[0, \infty)$.

$$
\begin{aligned}
\dot{V} & \leq-\theta_{2, \min }\|s\|_{2}^{2}-\frac{1}{n} \theta_{3, \text { min }}\|s\|_{2}^{4}+\|s\|_{2}\left(\beta_{1}+\beta_{2} l_{3}+\beta_{3} l_{3}^{2}\right) \\
& -\frac{1}{2} \sigma \sum_{i=1}^{n}\left(\hat{\theta}_{i_{-} 1}-\theta_{i_{-}}^{*}\right)^{2}+\frac{1}{2} \sigma \sum_{i=1}^{n} \theta_{i_{-}}^{* 2} \\
& -\theta_{i, \text { min }}^{*}\|s\|_{2}-\sum_{i=1}^{n} \varepsilon_{i}
\end{aligned}
$$

From both Case 1 and 2, we can conclude that because of sufficiently large $\theta_{1, \min }^{*}$ we obtain

$$
\dot{V} \leq-\theta_{2, \min }\|s\|_{2}^{2}-\frac{1}{2} \sigma \sum_{i=1}^{n}\left(\hat{\theta}_{i_{-1}}-\theta_{i_{-1}}^{*}\right)^{2}+d_{0}
$$

where

$$
d_{0}=\frac{1}{2} \sigma \sum_{j=1}^{n} \theta_{i-1}^{* 2}
$$

Because

$$
\beta=\min \left\{\frac{2 \theta_{2,2 x}}{\mu_{*}}, \frac{\sigma}{\left(\gamma_{1}^{3}\right)-}\right\}
$$

where $\left(\gamma_{1}^{-1}\right)_{\max }=\max \left\{\gamma_{1_{-}}^{-1}, \ldots, \gamma_{n_{-}}^{-1}\right\}$, it follows that for (24) become

$$
\dot{V} \leq-\beta V+d_{0}
$$

for $t \in[0, \infty)$, which implies that

$$
V(t) \leq\left[V(0)-\frac{d_{0}}{\beta}\right] \exp (-\beta t)+\frac{d_{0}}{\beta}
$$

Consequently, $s(t)$ and $\hat{\theta}_{i_{-}}(t), i=1, \ldots, n$, are ultimately bounded over for $t \in[0, \infty)$ and, furthermore, $s(t)$ will converge to the residual set $\Omega_{s}$ given by

$$
\Omega_{s}=\left\{s \in R^{n} \mid\|s\|_{2}^{2} \leq \frac{2 d_{0}}{p s_{n}}\right\}
$$

such that $e(t)$ and $\dot{e}(t)$ will also converge to a residual set whose size can be reduced by larger $k_{c, \text { nin }}$ and $\gamma_{1, \text { min }}$.

Note that the nonlinear term in (13) ensure that the closed-loop system is globally stable.

\section{Simulation Results}

In order to demonstrate the performance of the proposed adaptive decentralized controller, several numerical results are provided now. A control of two-link planar robot manipulator, shown in Fig. 1, is considered here. The manipulator is assumed to move on a horizontal plane and contact with the environment modeled as a straight line on the plane. The links are of uniform density and have the same length $l=1$. The masses of the links are $m_{1}=3$ and $m_{2}=1$, respectively. The gravitation is neglected in this study. The desired trajectory $q_{d}(t)=\left[q_{d 1}(t), q_{d 2}(t)\right]^{\top}$ is the time-varying vector-valued function defined by $q_{d 1}(t)$ $=1 \sin t, q_{d 2}(t)=1 \sin t$.

The numerical results of Theorem 2 are studied here. There is no friction in this case. The initial conditions used in the numerical study are $q_{1}(0)=q_{2}(0)=0, \dot{q}_{1}(0)$ $=\dot{q}_{2}(0)=0, \quad \hat{\theta}_{1-1}(0)=\hat{\theta}_{2_{-} 1}(0)=0$. For practical 405 implementation, the adaptive law with the $\sigma$-modification 
plementation, the adaptive law with the $\sigma$-modification term is considered. The control gains, adaptive gain constants, and auxiliary signals are, respectively, defined by use of $\lambda_{1}=\lambda_{2}=6, \theta_{1-2}=\theta_{2 \_2}=50, \theta_{1 \_3}=\theta_{2-3}=1, \gamma_{1}=\gamma_{2}$ $=0.5$, and $\sigma=0.5$. Figures 2 and 3 show the position profile of link 1 and 2, respectively, whereas Figures 4 and 5 represent the velocity profile of link 1 and 2 , respectively. From this figures, it is found that the position and velocity tracking errors approach to a residual set as time approaches infinite. Figures 6 and 7 demonstrate the updated parameters due to the adaptive law. It is represented that the parameters updated are uniformly bounded. Furthermore, Figures 8 and 9 illustrate that the two control inputs under this adaptive scheme are also bounded.

\section{Conclusion}

In this paper, we develop a new decentralized control scheme of robot manipulator for trajectory tracking. Due to nonlinear terms in the control law, the closed-loop system is globally stable. For numerical noise caused by the convergence of the time-varying layer, the adaptive law can be modified with a $\sigma$-modification term so that the tracking error will converge to a residual set whose size can be reduced by use of a proper design parameters. Therefore, it is suitable for implementation. Finally, a numerical study is provided to confirm the effectiveness of the proposed scheme.

\section{References}

[1] A. F. Filippov, "Differential equation with discontinuous right hand side," American Mathematical society Translations, vol. 42, no. 2. pp.199-231, 1964.

[2] L.-C. Fu, "Robust adaptive decentralized control of robot manipulators," IEEE Trans. Automat. Contr, vol. 37, no. 1, pp. 106-110, 1992.

[3] S.-H. Hsu and Li-Chen Fu, "Globally Fully Adaptive Decentralized Control of Robot Manipulator, To be submitted to Conference on Decision and Control, 2002.
[4] P. A. loannou and J. Sun, Robust Adaptive Control. Prentice-Hall, New Jersey, 1995.

[5] M. Liu, "Decentralized control of robot manipuiators: Nonlinear and adaptive Approaches," IEEE Trans. Automat. Contr., vol. 44, no. 2, pp. 357-363, 1999.

[6] M. Spong and M. Vidyasagar, Robot Dynamics and Contorl, Wiley, New York, 1989.

[7] Y. Tang, M. Tomizuka, G. Guerrero, and G Montemayor, "Decentralized robust control of mechanical systems," IEEE Trans. Automat. Contr, vol. 45, no.4, pp.771-776, 2000.

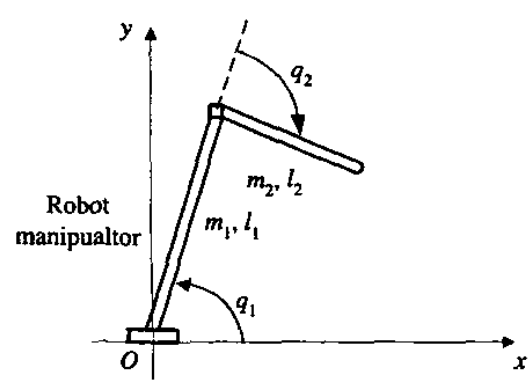

Fig. 1 Two-link planar robotic manipulator

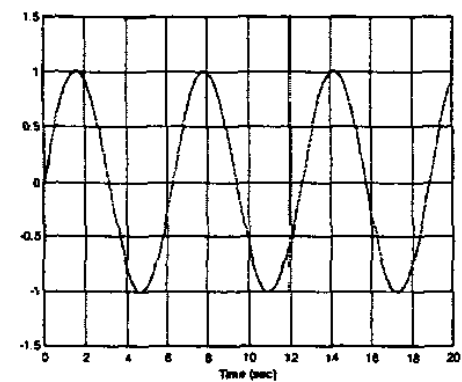

Figure 2. Position trajectory of Link 1

$$
\text { ( } q_{1}: \text { “-” line, } q_{d 1}: \text { “.." line) }
$$




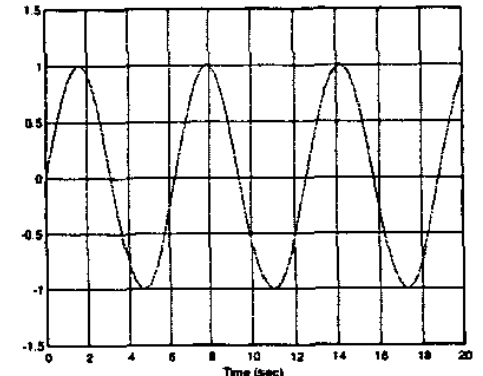

Figure 3. Position trajectory of Link 2

( $q_{2}:$ :-" line, $q_{d 2}:$ “.." line)

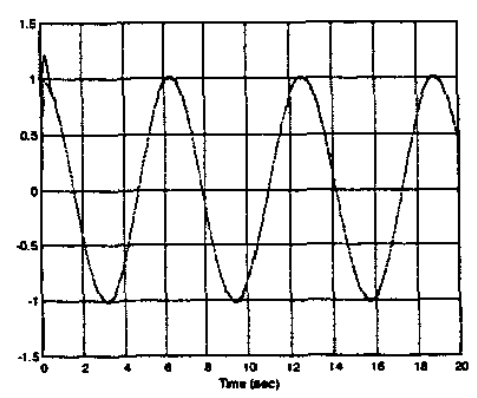

Figure 4. Velocity trajectory of Link 1

$$
\text { ( } \dot{q}_{1}: \text { "-" line, } \dot{q}_{d 1}: \text { "..." line) }
$$

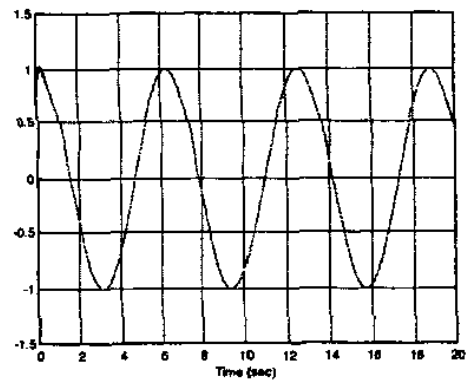

Figure 5. Velocity trajectory of Link 2

( $\dot{q}_{2}:$ "_, line, $\dot{q}_{d 2}:$ "..." line)

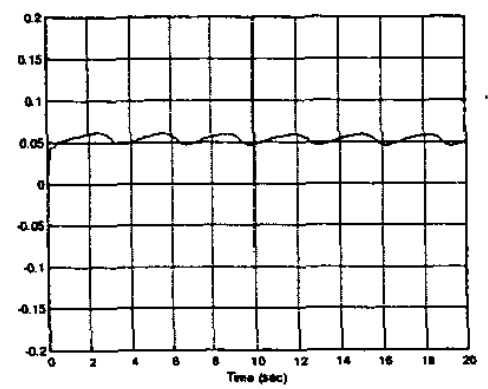

Figure 6. Estimated parameters $\hat{\theta}_{1,1}$ of Link 1

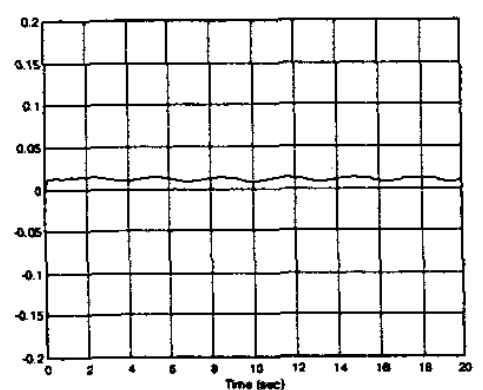

Figure 7. Estimated parameters $\hat{\theta}_{2_{-}}$, of Link 2

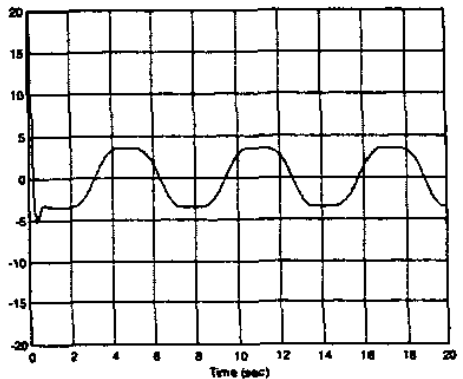

Figure 8. Control input $\tau_{1}$ of Link 1 .

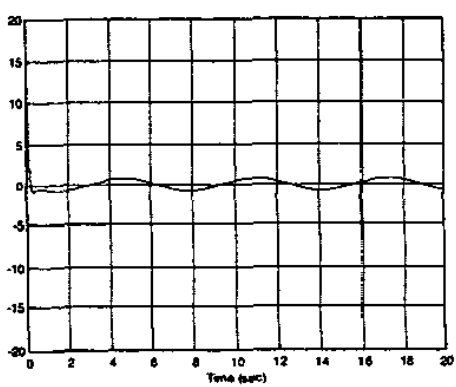

Figure 9. Control input $\tau_{2}$ of Link 2 . 